\title{
Properties of the Liquefied Natural Gas Waterway terminals Networks based on the Betweenness Centrity
}

\author{
Di Cui \\ Research Associate, China Waterborne Transport Research Institute, Safety and Emergency Research Division \\ No.8 Xitucheng Road, Haidian District, Beijing, 100088 \\ cuidi@wti.ac.cn
}

\begin{abstract}
The method analysis the topological properties of Liquefied Natural Gas Waterway terminal Networks is studied based on the betweenness centrity in this paper. LNG shipping systems are vital to the economic development of our country. And it is critical for Chinese economic growth. Furthermore, the Chinese liquefied natural gas waterway terminal network hub degree is combined by the local economic growth. Especially, the shipping volume, degrees, weights characteristics and modular properties are mainly studied in Chinese Liquefied Natural Gas Waterway terminal Networks. This research on Liquefied Natural Gas Waterway terminal hub degree and weight characteristics can improve economic benefits and the hub waterway terminals location. Here, the betweenness centrity of LNG shipping volume information is used during the year 2010-2018. Furthermore, we analysis the network of links between liquefied natural gas waterway terminals with the rationality. The network has several features that set it apart from other transportation networks are shown.
\end{abstract}

Keywords-Waterway terminal Networks, the networks properties, Liquefied Natural Gas Waterway terminal, Betweeness Centrity

\section{INTRODUCTION}

Liquefied natural gas is crucial to develop of Chinese industry. Furthermore, they played the great part indicators for the economic and development. Natural gas as an efficient, clean and renewal energy. Liquefied natural gas systems affect the industry, the national economy and people of daily life. Approximately $90-95 \%$ of international liquefied natural gas goods are long-haul transported by the sea because of shipping cost efficiency. Shipping can get a discount on sailing the liquefied natural gas all over the world. Especially, nowadays more and more LNG waterway terminal are constructed nowadays. During the past time, the network topological properties analysis has been used to study networks systems. In this paper, we study the statistical properties of the liquefied natural gas waterway terminal networks. The nodes stands for the waterway terminals and the links are the LNG transportation connecting the waterway terminals. Based on two different representation of network topology. The hub LNG waterway terminals are special waterway terminals that service as transshipment and switching LNG systems. The hub facilities concentrate flows in order to take advantage of economies of scale. Flows from the same origin with different destinations are consolidated on their route to the hub and are combined with flows that have different origins but the same destination. The consolidation is on the same route from the origin LNG ship waterway terminal to the LNG hub waterway terminals, such as Tianjin LNG waterway terminal and from the hub to the other destination waterway terminal as well as between LNG hubs waterway terminal.

The hub LNG waterway terminal location problem is concerned with locating hub facilities and allocating LNG demand nodes to hubs waterway terminals between origindestination pairs. They different in how non-hub nodes are allocated to the LNG hubs waterway terminals. In single LNG waterway terminal allocation, all the incoming LNG shipping volume and outgoing LNG shipping volume of economic demand is routed through a single LNG waterway terminal hub. The allocation aspect of LNG hub location the problems are studied. But since optimal allocations are affected by LNG waterway terminal hub locations and optimal hub locations are affected by the LNG volume location for the liquefied natural gas waterway terminals networks. Theoretical properties of the LNG waterway terminals description of the formation is far from being developed well.

In this paper, we present an investigation of the Chinese liquefied natural gas waterway networks (CLNGWN). Recently, Péter Pollner a.et al. (2002) provided a brief review of the Probabilistic description of traffic breakdowns networks. Later, Martin L. Hazelton and David P. Watling (2004) discussed the computation of equilibrium distributions of traffic-assignment models. Fernando González Laxe a, Maria Jesus Freire Seoane b, Carlos Pais Montes b (2012) discussed the maritime degree, centrality and vulnerability: port hierarchies and emerging areas in containerized transport in 2008-2010. Here, we consider the Chinese Liquefied Natural Gas Waterway terminal Networks (CLNGWTN), which comprises 28 waterway terminals in different locations. We have gathered the ship schedule information from the Internet. The nodes of the network are the waterway terminals and the edges are the lines connecting them along the various volume LNG shipping route.

The LNG shipping networks with the scale-free properties is generated with various methods. The topological of the scale-free model are identified as being necessary for the Liquefied natural gas network to have the scale-free property. The LNG network grows with the preferential attachment properties and its nodes with a large 
degree are more likely to create links to new waterway terminals nodes than ones with the small amount degree.

The rest of this paper is organized as follows. Section 2 gives the LNG networks problem description and notation definition. Section 3 proposes LNG scale-free properties, computer simulation and analysis results of the Chinese liquefied natural gas waterway terminal networks (CLNGWTN). Furthermore, the numerical experiments based on the Chinese liquefied natural gas waterway terminal networks (CLNGWTN) are carried out in China. Finally, the conclusion is given in Section 4.

\section{NOTATION AND PROBLEM DESCRIPTION}

\section{A. The Chinese liquefied natural gas waterway terminal networks}

We will implement different scenarios by considering different $\mathrm{A}$. We assume the Chinese liquefied natural gas waterway terminal networks (CLNGWTN) considered in this paper are built, owned and operated by citizens of $\alpha$ country A. The set of waterway terminals, which can be further classified into two disjoint subsets: the set of hub waterway terminals denoted by PC and the set of feeder waterway terminals PF. The maritime cabotage legislations in liner $\mathrm{H} \& \mathrm{~S}$ shipping network design can be simply described by introducing an indicator for each pair of waterway terminal $i$ and terminal $j(i j \in P$ ). If ships of country A are allowed to directly transport containers from waterway terminal $i$ to waterway terminal $j$; cij is equal to 1 ; otherwise cij is 0 of Chinese liquefied natural gas waterway terminal networks (CLNGWTN).

\section{B. The directed network of the Chinese LNG shipping volume}

The Chinese LNG shipping network of the entire LNG fleet is noticeably asymmetric, with $82 \%$ of all linked OD volume pairs of waterway terminals being connected. Still, the majority of waterway LNG terminals belongs to the single strongly connected component, i.e. for any two waterway terminals in this component there are routes in both directions, though possibly visiting different intermediate ports. The routes are intriguingly short: only few steps in the network are needed to get from one port to another. The shortest path length between two waterway terminals is the minimum number of nonstop connections one must take to travel between origin and destination.

The structure of Chinese LNG waterway terminal networks can be symbolized by an asymmetrical weight matrix W whose weight element $W_{i j}$ is the number of LNG transport liners traveling from waterway terminal $i$ to waterway terminal $j$. We should note that the weight element $W_{i j}$ includes the contribution from the direct ship transportation between the terminals $i$ and $j$ without middle stop seaports.

The weights $W_{i j}$ denotes the link $(i j)$ when the LNG demand flow, and $\mathrm{w}^{1}$ ij assumed randomly in the range $(0,1]$ for each link in our simulations.

First, we employ $\mathrm{k}$ to denote the degree of a given node $\mathrm{i}$ for directed Chinese LNG waterway terminal networks to represent the undirected degree of the Chinese LNG waterway terminal networks with the same structure with scale-free topology. The degree of waterway terminal i stand for number of waterway terminals. The Chinese LNG waterway terminal networks consists of nodes representing waterway terminals and links between two nodes exists if they are consecutive stops on the route with the traffic volume. The node degree $\mathrm{k}$ in this topology is just the number of different shipping routes one can take from a given waterway terminal. An edge between two nodes means that there is a ship schedule traveling between them. From which one can arrive at waterway terminal $i$ and the number of waterway terminals that can be reached from waterway terminal i, respectively. Using the weighted matrix, we can write these quantities as Equation (1)-(3).

$$
\begin{gathered}
\text { ki=Cijwij Eij } \\
\mathrm{Si}=\text { aibj } \sum_{\mathrm{i}-1}^{\mathrm{j}} \text { wij dij. } \\
\mathrm{Eij}=1 / \mathrm{N}(\mathrm{N}-1) 1 / \mathrm{dij}
\end{gathered}
$$

Where Cij is a unit step function, ai and bj are the coefficients, which takes 1 for $x>0$ and 0 otherwise. If dij is the distance between the waterway terminals $i$ and $j$, the decline in mutual interaction is expressed in terms of a distance deterrence function $\mathrm{Si}=$ aibj $\sum_{\mathrm{i}-1}^{\mathrm{j}}$ wij dij.

Similarly, we can also obtain the above equations. As the Chinese LNG waterway terminal network is a directed connected network with 28 nodes standing for the LNG terminals, we report the size of the giant strongly component defined. We find that the strongly connected component, i.e., every pair OD shipping volume of waterway terminals is connected in both directions, comprises 28 LNG terminals. The sizes of the component are found to be 28 nodes for the waterway terminal LNG networks. This indicates that the corresponding adjacency matrix for the network is almost symmetrical. Next, we consider the LNG demand flow of the weighted Chinese LNG waterway terminals networks. We define the total LNG demand traffic flow coming into node i as the strength.

The large amount of LNG shipping demand flow in both spaces suggests that database is highly redundant in the Chinese LNG topological scale-free structure; i.e., most connections between the OD shipping volume pairs of waterway terminals are represented by more than one ship. This makes the analysis of Chinese LNG network topology reliable.

\section{SIMULATION AND RESULTS}

In our simulations, the network topologies considered here are the lattice networks as the example of regular networks, random networks generated by Chinese liquefied natural gas waterway terminal networks model (The rewiring probability is 0.1 here). The average degree $<\mathrm{k}>$ of the Chinese LNG 
waterway terminal networks is $<\mathrm{k}>=7$ (i.e., $\mathrm{m}=4$ ), as it resembles typical crossroads. The size of the generated network varies from 86 to 92 . All the discussed resulting data is averaged more than 20 realizations.

An unusual measure for the investigated the performance of Chinese liquefied natural gas waterway terminal networks (CLNGWTN) can be characterized. Numerically investigated the Chinese liquefied natural gas waterway terminal networks (CLNGWTN) demand flows assignments over three ship types (container ships, the bulk ships and the LNG ships) topologies, it suggests that both the assignment strategy of Chinese liquefied natural gas waterway terminal networks (CLNGWTN) and the sale-free topology are significantly for the bulk cargo, the container and the LNG demand shipping flows volume (the node degree) distribution, as shown in Fig. 2. All the discussed resulting data is averaged more than 20 realizations.

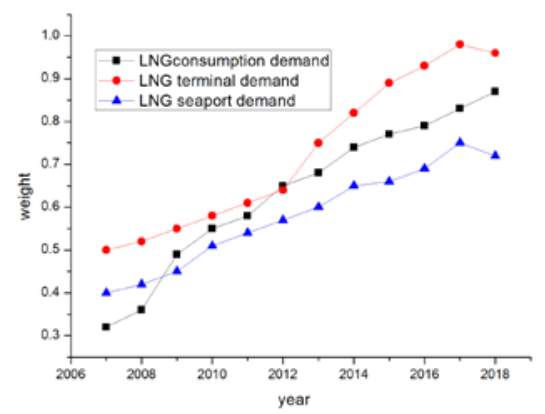

Figure 1 The relationship of weight for the LNG consumption demand, the LNG terminal demand and the LNG seaport demand in the Chinese liquefied natural gas waterway terminal networks from the year 2006 to 2018(CLNGWTN).

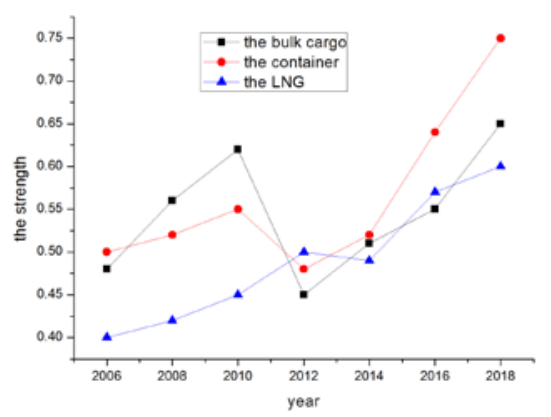

Figure 2 The relationship of demand strength for the Chinese liquefied natural gas waterway terminal networks from 2006 to 2018 about the bulk cargo, the container and the LNG.

Fig. 1 shows the relationship of weights of Chinese LNG waterway terminal networks from the year 2006 to 2018. From Fig.1, the vertical axis represents the weights and the horizontal axis, the year of the act-size. The solid cycles denote the empirical data for the LNG consumptions demand,

Fig. 2 shows the distribution of accumulative demand strength for CLNGWTN from the year of 2006 to 2018. In the Fig.2, the vertical axis represents the weights, and the horizontal axis, a certain number of the years (from 2006 to 2018). The solid cycles denote the empirical data. The solid line the least-square fitting.

Studying of Chinese LNG waterway terminal networks have triggered the tremendous interests nowadays. One of the scale-free characters of Chinese LNG networks can not only helping to study the scale-free topological structure, but also providing the new methods for its applications, such as database mining. We show that the LNG waterway terminal network has some other characters that setting it apart from other cargo transportation networks. In particular, LNG waterway terminal network has the scale-free characterizes. The network of all LNG ship movements possesses a heavy-tailed distribution for the waterway terminals and for the LNG loads transported. Waterway terminal plays an important role in the network topology of Chinese LNG networks. We also study the LNG flow of Chinese LNG networks, based on the weighted network representation, and demonstrate the weight distribution, which can be described by power law or exponential function depending on the assumed definition of network topology.

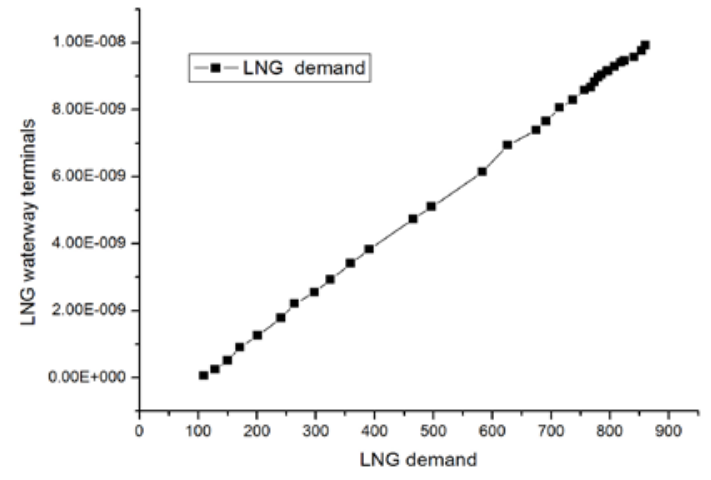

Figure 3 The relationship of LNG demand and the scale-free properties of the LNG waterway terminals in the Chinese liquefied natural gas waterway terminal networks from the year 2006 to 2018.

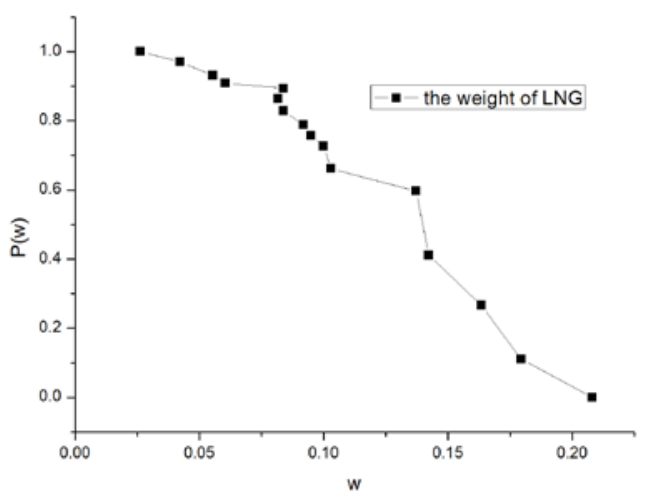

Figure 4 The relationship of weight $\mathrm{w}$ and $\mathrm{P}(\mathrm{w})$ in the Chinese liquefied natural gas waterway terminal networks from the year 2006 to 2018.

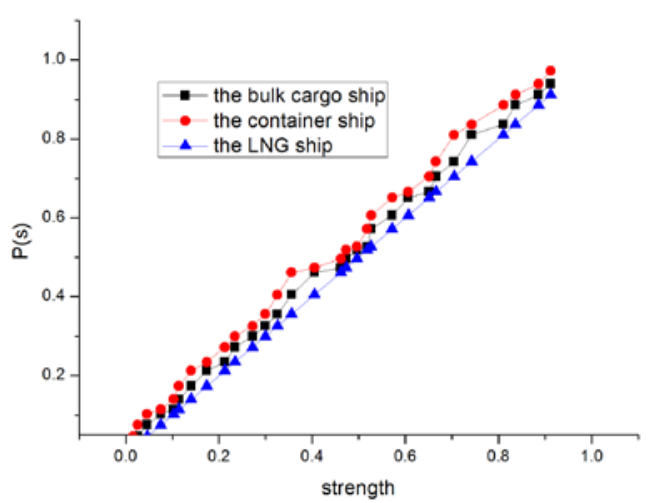

Figure 5 The relationship of weight $\mathrm{w}$ and $\mathrm{P}(\mathrm{w})$ for the bulk cargo ship, the container ship and the LNG ship in the Chinese liquefied natural gas waterway terminal networks from the year 2006 to 2018. 
In summary, we have studied the statistical topological properties of liquefied natural gas waterway terminal networks in China. We explore scaling laws and correlations that may govern intrinsic features of the network. The topological properties, including the degree distribution, weight distribution and strength distribution are studied. That LNG waterway terminals with high memberships play a critical role in three kinds of the networks. Furthermore, our investigations revealed interesting differences between the two types of networks concerning the dependence of the betweeness centrality measures on the relative out-degree of community members (the ratio of out-degree versus number of all nearest neighbors within the community).

\section{CONCLUSION}

In conclusion, we have analyzed the statistical properties of Chinese LNG waterway networks. We explore scaling laws and correlations that may govern intrinsic features of such network. The topological properties, including the degree distribution, weight and strength are studied. Our results indicate that nodes with high memberships play a central role in these networks. Furthermore, our investigations revealed interesting differences between the two types of networks concerning the dependence of the centrality measures on the relative out-degree of community members (the ratio of out-degree versus number of all nearest neighbors within the community).

\section{ACKNOWLEDGMENT}

This paper is an effort product of the projects of Chinese
Safety Committee of waterway terminal. The writer is grateful for the technical assistance provided by all committee members. This work is financially supported by the Chinese Safety Committee of waterway terminal under Grant Nos.410X01 and Nos.41201.

\section{REFERENCES}

[1] Reinhart Ku "hne, Reinhard Mahnke, Ihor Lubashevsky, Probabilistic description of traffic breakdowns. Physical Review E, Volume 65, 066125, 2002.

[2] Péter Pollner a, Gergely Palla a, Dániel Ábel b, András Vicsekc, Illés J. Farkas a, Imre Derényi b, Tamás Vicsek Centrality properties of directed module members in social networks, Physica A 387,49594966,2008

[3] L. C. Freeman. Centrality in social networks conceptual clarification. Social Networks, 1(3) (1979),215 - 239.

[4] T. Erez, M. Hohnisch, and S. Solomon. Economics: Complex Windows, chapter Statistical Economics on Multi-Variable LayeredNetworks, page 201 , (2005)Springer.

[5] Bell, M. G. H., Liu, X., Angeloudis, P., Fonzone, A., \& Hosseinloo, S H.. A frequency-based maritime container assignment model. Transportation Research Part B, 45(5) (2011)1152-1161.

[6] E. A. Leicht and M. E. J. Newman. Community structure in directed networks. Physical Review Letters, 100:118703, 2008.

[7] Hazelton, M. L.. Some remarks on stochastic user equilibrium. Transportation Res. 32B 101-108,1998.

[8] Hazelton, M. L.. Day-to-day variation in Markovian traffic assignment models. Transportation Res. 36B, 61-72,2002.

[9] Martin L. Hazelton and David P. Watling .Computation of Equilibrium Distribution of Markov Traffic-Assignment Models. Transportation Science.331-342,2004. 